REGARDS

SUR LECONOMIE ALLEMANDE

BULLETIN ECONOMIQUE DU CRAC

\section{Regards sur l'économie allemande}

Bulletin économique du CIRAC

109 | 2013

Varia

\title{
" Justice sociale ». Le modèle social allemand en question
}

Analyse

Isabelle Bourgeois

\section{(2) OpenEdition}

Journals

Édition électronique

URL : http://journals.openedition.org/rea/4560

DOI : $10.4000 /$ rea.4560

ISSN : 1965-0787

Éditeur

CIRAC

Édition imprimée

Date de publication : 4 juillet 2013

Pagination : 35-38

ISSN : 1156-8992

\section{Référence électronique}

Isabelle Bourgeois, « « Justice sociale ». Le modèle social allemand en question », Regards sur l'économie allemande [En ligne], 109 | juillet 2013, mis en ligne le 19 juillet 2013, consulté le 15 septembre 2020. URL : http://journals.openedition.org/rea/4560

Ce document a été généré automatiquement le 15 septembre 2020

(c) CIRAC 


\section{« Justice sociale ». Le modèle social allemand en question}

Analyse

Isabelle Bourgeois

1 Pauvreté, "dumping salarial», injustice sociale... seraient le prix payé par les Allemands pour la compétitivité de leur économie. Voilà la nouvelle 'doxa' française depuis un an, à en croire la plupart des économistes ou journalistes et même certains responsables politiques. L'instrumentalisation populiste des arguments de campagne avancés par l'opposition allemande à la veille des élections au Bundestag (le 22 septembre) bat son plein... en France. La revendication centrale dans le contexte allemand - "plus de justice sociale» - est utilisée en France pour construire l'épouvantail d'une «Allemagne anti-sociale » dans le but de sanctuariser un «modèle social français » qu'il conviendrait pourtant de réformer d'urgence pour en assurer le financement pérenne, et de nourrir un tir de barrage défensif contre, alternativement, «l'intransigeance égoïste " d'une Angela Merkel ou une «Bruxelles » à la fois trop libérale et trop interventionniste.

2 Les mêmes " éléments de langage » (paupérisation, précarisation, démantèlement du modèle social...) n'ont pourtant ni le même sens ni la même finalité des deux côtés du Rhin. En Allemagne, le débat sur la « justice sociale » relève de la normalité des règles en usage durant une campagne électorale, et ce débat est pluraliste par définition; l'instrumentalisation qui est faite par certains de cas de dysfonctionnement isolés peut être corrigée par des positions contraires, et surtout par une réflexion collective axée sur les faits. En France, ces éléments servent à nourrir dans une sphère politicomédiatique avide du monopole d'opinion un mainstream ne souffrant aucune contradiction, ni encore moins la confrontation avec la réalité des faits.

\section{Le 'débat' français : haro sur le modèle allemand}

3 Pour le dire autrement: en Allemagne, ces questions nourrissent un débat public où s'affrontent des perspectives politiques et scientifiques différentes justifiant des 
analyses divergentes, $\mathrm{y}$ compris dans les médias. Le plus important dans ce contexte est que le "d'où parle » chacune des voix impliquées est connu de tous, ce qui contribue à la transparence démocratique. En France, la donne est tout autre : d'abord, la réalité débattue est celle d'un autre pays, et elle est même souvent totalement étrangère à ceux qui dissertent sur elle, ce qui les expose à la réception comme à la diffusion de contre-vérités issues d'Allemagne. Dès lors, les pièges qui se tendent aux observateurs français sont aussi nombreux que classiques: on se rassure sur la véracité de la perspective propagée en l'étayant de données macro-économiques modélisées et internationalisées (taux de pauvreté, par exemple) ; pour respecter la couleur locale, on se fonde sur des statistiques allemandes - mais sans en connaître le contexte ni l'instrumentalisation qui peut en être faite outre-Rhin -, ou alors on se borne à puiser dans celles des milieux syndicaux dont les chiffres sont par réflexe jugés plus véridiques que ceux de toutes les autres institutions, aussi indépendantes qu'elles puissent être -; on recourt à des témoignages ou à des cas isolés qu'on connait personnellement pour enraciner dans le réel la construction de l'analyse d'une réalité étrangère hypothétique et lui conférer cette touche humaine donnant l'illusion du " parler vrai ». Et comme nous sommes socialisés chacun dans un contexte culturel (et socio-culturel) spécifique, nous nous servons de nos propres repères pour les appliquer à une réalité difficile à cerner, ce qui nous rassure, mais cultive notre presbytie.

\section{Amalgame et désinformation...}

L'universalisme de la pensée française, doublé de l'habitude encore faiblement développée chez nous de l'observation des réalités étrangères, nous incite tout particulièrement à tomber involontairement dans ces pièges - quand nous ne le faisons pas de propos délibéré. Mais alors, c'est aux consommateurs de nos analyses que nous tendons ces pièges, sachant pertinemment qu'ils ignorent encore plus qu'un expert et la donne et les enjeux des débats du pays voisin. Plus le sujet est complexe, plus il suffit de quelques rares chiffres pour fausser une réalité : un pourcentage donné sans valeur de référence, par exemple, un salaire horaire cité sans que soit évoquée au moins la branche où il est en usage ou le type d'emploi et de qualification auquel il correspond de telles pratiques favorisent non seulement l'amalgame, elles appartiennent aussi au répertoire classique de la désinformation et du discours partisan. En campagne électorale, les Allemands ne s'en privent pas non plus; la seule différence, c'est que, eux, disposent généralement des clés pour décrypter, ce dont se charge normalement une presse pluraliste.

\section{... dans un but de 'contre-propagande'}

5 Les fausses allégations et contre-vérités sur le «modèle allemand " et ses «mythes » sont d'autant plus prisées dans une France en perte de compétitivité, confrontée à un fort chômage structurel et aux prises avec des doutes existentiels, que les réformes structurelles menées en Allemagne par le gouvernement Schröder il y a dix ans ont contribué à permettre à l'économie allemande de renouer avec la compétitivité et à générer des emplois. Mais comme certaines d'entre elles ont accru la flexibilité du travail, via notamment l'extension du segment des bas salaires, elles doivent impérativement être présentées comme "anti-sociales » dans une France idéalement égalitaire où la perspective de la « flexi-sécurité » inquiète au plus haut point. 
Parmi l'abondante production actuelle d'anti-modèles allemands dans les médias et l'espace public français, l'ouvrage "Made in Germany. Le modèle allemand au-delà des mythes » révèle avec une rare limpidité la finalité de cette nouvelle doxa française. Dans son introduction, Guillaume Duval affirme en effet: "...- c'est en tout cas la thèse de ce livre - que l'action de Gerhard Schröder ait plutôt fragilisé à terme l'économie et la société allemandes en permettant que s'y répandent la pauvreté et les inégalités... ", avant d'avouer: "... ce qui est particulièrement frappant dans les débats actuels [français; IB], c'est de constater combien le 'modèle allemand' fait l'objet d'une instrumentalisation propagandiste qui n'a que faire des réalités. Cette mauvaise foi des thuriféraires du 'modèle allemand' ne peut cependant fonctionner que parce qu'elle s'appuie sur l'ignorance profonde... des Français au sujet de l'Allemagne». Il convient donc de "donner au public français des clés de compréhension... Ce qui contribuera, je l'espère, à sortir enfin le débat sur le 'modèle allemand' de l'instrumentalisation où veulent l'enfermer ceux qui mènent avec tant d'ardeur le combat en faveur du moins-disant social en France et en Europe». On ne saurait être plus clair : le propos de cet ouvrage - comme de tant d'autres prises de position qui font florès en ligne et off line - est 'contre-propagandiste'. Et dès lors, la touche humaine ne peut faire défaut : en l'occurrence, l'amitié de l'auteur avec un Allemand qui, « aujourd'hui chômeur de longue durée et soumis aux contraintes de la loi Hartz IV,... est, malheureusement, une des nombreuses victimes » des réformes Schröder; c'est là, ajoute-t-il dans sa conclusion, "une raison plus personnelle de m'insurger contre l'image très positive (mais fausse) qu'on donne en France actuellement de cette politique».

\section{Les principales lignes du débat en Allemagne}

Qu'en est-il réellement? Dans l'espace public allemand aussi, les réformes Hartz sont un sujet sensible (voir REA 108/2013) et constituent une des principales lignes de clivage du débat public. Se trouvent particulièrement sur la sellette : une disposition de la Loi Hartz II qui assouplit le régime des petits boulots à $450 €$ (" mini-jobs»); et la Loi Hartz IV qui crée un régime d'assistance à deux volets (soutien pécuniaire + programme de réinsertion/requalification) pour les demandeurs d'emploi ne pouvant prétendre aux allocations de l'assurance chômage et prévoit également des aides destinées à ses proches vivant sous son toit. Les analyses publiées en Allemagne quant à leur impact sur l'emploi ou la richesse/pauvreté sont rarement neutres. Il faut dire que ces effets se laissent difficilement mesurer, tant l'interaction des multiples facteurs à prendre en considération est complexe: globalisation, mutations de l'économie et de l'emploi depuis les années 1990 (tertiarisation...), diffusion des TIC, hausse généralisée des qualifications, évolution des modes de vie et des valeurs (individualisation, éclatement du modèle familial, progression de l'emploi féminin, flexibilité de plus en plus souvent choisie des biographies professionnelles...).

\section{Deux perspectives scientifiques : ...}

S'ajoute à cela la coexistence de deux perspectives disciplinaires dans le champ scientifique, qu'on interprète trop hâtivement comme idéologiques en France. D'une part celle des sociologues qui considèrent la réalité sociale sous l'angle de l'inclusion et de la lutte contre la pauvreté (et qui ont de ce fait parfois tendance à 'victimiser' ceux qui exercent un mini-job ou perçoivent les aides au titre de Hartz IV) ; ils se recrutent 
notamment dans la mouvance syndicale (syndicats, centres de recherche comme l'IMK ou l'IZA) et partiellement au sein de l'institut IAB de l'Agence pour l'emploi de Nuremberg dont la mission est d'explorer les deux problématiques à la fois. D'autre part, celle des économistes qui abordent ces questions sous l'angle des mutations du marché de l'emploi ou du financement de la protection sociale, et qui aboutissent dès lors au constat d'une meilleure adéquation entre offre et demande sur un marché du travail qui continue à créer des emplois malgré la récession, ou d'une consolidation des comptes sociaux qui garantit à moyen terme l'avenir du financement du modèle social allemand sans mettre en péril la compétitivité économique. Ils se recrutent parmi les grands instituts de conjoncture, sans exclure le Conseil des Sages qui conseille en toute indépendance tout gouvernement fédéral dans la définition de sa politique générale, ou encore dans les milieux proches de l'industrie (Institut der deutschen Wirtschaft) dont la principale préoccupation est le développement de la compétitivité, source de croissance et donc d'emploi.

\section{... ne pas relâcher la 'discipline sociale' versus protection des acquis sociaux}

Ces deux approches mènent à deux positions opposées dans le débat public: l'une plaide pour ne pas revenir en arrière sur la réforme de l'Etat social et la flexibilité, refusant de relâcher la 'discipline sociale' ; l'autre revendique le retour à une logique de protection des acquis sociaux et à plus de redistribution. Dans ce dernier cas, l'argument selon lequel la multiplication des mini-jobs mènerait à la précarisation générale de l'emploi et serait source de paupérisation sert alors à étayer la revendication d'un revenu "socialement souhaitable " sous la forme d'un SMIC légal généralisé ou d'une révision des lois Hartz - mais sans que jamais cette problématique soit replacée dans le contexte économique global et sans que soit posée la question du financement (poids des prélèvements) de ce modèle social ni celle de son efficience pour la cohésion sociale (insiders/outsiders). Les publications des experts proches de la mouvance syndicale et de la gauche de l'opinion en donnent actuellement de nombreuses illustrations ; elles alimentent les programmes électoraux.

\section{Les faits : mutation des formes d'emploi et des comportements}

10 Extrêmement rares sont dès lors les analyses neutres et factuelles. Dans le numéro 4/2013 de la très réputée revue WSI Mitteilungen (soutenue par la Fondation Hans Böckler proche du DGB), par exemple, deux chercheurs dont Ulrich Walwei, directeur adjoint de l'IAB, dressent un état des lieux le plus objectif possible des travaux scientifiques étudiant l'impact sur le marché du travail des trois formes les plus classiques de l'emploi atypique: intérim, CDD et mini-jobs. L'analyse globale de l'évolution du marché du travail depuis 2005 révèle que "le socle structurel du chômage s'est réduit " mais que, parallèlement à cette embellie, on constate une "mutation des formes d'emploi » ou hausse de l'emploi atypique : en juin 2012, on recensait ainsi 7,4 millions de mini-jobs (dont 2,6 millions sont des emplois complémentaires), 2,7 millions de CDD et 900000 emplois intérimaires. Les «causes de cette évolution sont multiples » et résident avant tout dans "les mutations du comportement des acteurs économiques" en réaction à l'évolution du contexte (droit du travail, cotisations sociales, possibilité de concilier vies familiale et professionnelle...). Quant aux effets positifs ou négatifs sur la 
situation de l'emploi ou le marché du travail en général, il n'est pas possible, en l'état actuel des travaux, de tirer une conclusion univoque. Néanmoins, si la rémunération de l'emploi atypique est inférieure à celle de l'emploi régulier et si les risques de chômage sont plus élevés, certains effets sont indubitablement positifs : comme «ils évitent la dévalorisation du capital humain, ils réduisent en tendance le risque d'enracinement dans le chômage »; le bénéfice est psychologique aussi, puisque l'activité est source d'inclusion sociale, ce qui accroît l'autonomie des individus. Au total, «ces effets positifs d'un accès plus facile à l'emploi se diffusent également dans d'autre domaines de la société».

\section{CDD = période d'essai $;$ mini-jobs = boulots d'appoint}

11 Si on considère l'emploi atypique sous l'angle de la transition vers l'emploi régulier, le "tableau est contrasté». Ainsi, les CDD remplissent souvent "la fonction d'une période d'essai élargie » ménageant la transition vers un emploi régulier, surtout pour les actifs débutants et les actifs hautement qualifiés. Mais ils accroissent aussi le risque de chômage, particulièrement pour les moins qualifiés, qui sont embauchés en CDD plutôt pour des raisons de flexibilité. Dans l'intérim cette fonction de transition est moins nette (à la différence de la France) que pour les CDD. Et dans le cas des mini-jobs, la question ne se pose gère, puisqu'ils sont majoritairement choisis "pour apporter un revenu complémentaire au ménage ou comme boulot d'appoint" par des épouses, des retraités et des étudiants. Si on considère le marché du travail, alors on constate que "les formes d'emploi atypique remplissent une fonction de protection des salariés en CDI en permettant d'amortir les fluctuations du marché». C'est surtout l'intérim qui joue ce rôle d'outil de flexibilité (et d'indicateur précoce des variations conjoncturelles), suivi des $\mathrm{CDD}$. Des effets de substitution à l'emploi régulier ne sont pas décelables, encore moins dans le cas des mini-jobs qui se sont, eux, plutôt substitués au travail au noir.

\section{Activer l'ascenseur social par la hausse des qualifications}

Globalement, s'il est devenu beaucoup plus facile de trouver un emploi en Allemagne, le marché du travail s'est segmenté, reléguant à la marge (mais sans les exclure) les moins qualifiés des actifs. Et dès lors, la politique la plus adaptée pour résoudre le problème de cette marginalisation croissante réside dans la prévention: outre une politique générale de réforme de la fiscalité des ménages ou de mesures permettant de mieux concilier vies familiale et professionnelle, elle implique de rendre plus efficient encore le système d'éducation et de formation professionnelle, initiale et continue, afin de créer les conditions nécessaires au bon fonctionnement de l'ascenseur social. C'est cette approche qui fait consensus en Allemagne - $a$ fortiori au sein du mouvement syndical. Incriminer les mini-jobs comme une «trappe à bas salaires» (argument notamment de la Confédération syndicale DGB) relève principalement de la rhétorique électoraliste. (IB) 
INDEX

Mots-clés : politique sociale, état social, modèle social, relations franco-allemandes,

comparaison franco-allemande, société civile, protection sociale, marché du travail, emploi 